\title{
La Ley 11/2007, de Acceso Electrónico de los Ciudadanos a los Servicios Públicos y el uso del software libre en la Administración Pública
}

\author{
Diana DEL VALLE GARCÍA \\ Archivera y Bibliotecaria de la Autoridad Portuaria de Baleares \\ dvalle@portsdebalears.com
}

Recibido: Diciembre 2012

Aceptado: Febrero 2013

Resumen: El presente trabajo analiza el software libre en relación con la Ley 11/2007, de Acceso Electrónico de los Ciudadanos a los Servicios Públicos. Para ello se parte de un estudio de la legislación española vigente y de las directivas europeas y su relación con la aplicación del software libre en la Administración Pública española.

Palabras clave: Administración, Administración electrónica, derechos del ciudadano, Ley 11/2007, licencias de software, obligaciones de la Administración, software libre.

\section{The Law 11/2007, of Electronic Access of the Citizens to the Public Ser- vices and the use of the free software in the Public Administration.}

\begin{abstract}
The present work analyzes the free software in relation with the Law 11/2007, of Electronic Access of the Citizens to the Public Services. For it, it splits of a study of the Spanish in force legislation and of the European boards and his relation with the application of the free software in the Public Spanish Administration.
\end{abstract}

Keywords: Administration, electronic Administration, rights of the citizen, Law 11/2007, licenses of software, obligations of the Administration, free software.

Abreviaturas: CENATIC: Centro Nacional de Referencia de Aplicación de las Tecnologías de la Información y de la Comunicación.

GPL: Licencia Pública General.

LPAC: Ley 30/1992, de 26 de noviembre, de Régimen Jurídico de las Administraciones Públicas y del Procedimiento Administrativo Común.

LPI: Ley de Propiedad Intelectual.

RPSEC: Real Decreto 772/1999, de 7 de mayo, que regula la presentación de solicitudes, escritos y comunicaciones ante la Administración General del Estado, la expedición de copias de documentos y la devolución de originales, así como el régimen de las oficinas de registro.

TIC's: Tecnologías de la Información y del Conocimiento.

TCO: Total Cost of Owership. 


\section{INTRODUCCIÓN: OBJETIVOS Y METODOLOGÍA}

La aplicación de la Ley 11/2007, de Acceso Electrónico de los Ciudadanos a los Servicios Públicos y el establecimiento de garantías para el acceso de los ciudadanos pueden ser elementos convergentes mediante la implantación del software libre en la Administración. Mediante el análisis y estudio de las licencias libres y de la citada Ley se pretende demostrar la conveniencia de la aplicación y del uso del de las mismas en la Administración Pública española.

De esta manera, los objetivos del presente trabajo se pueden enumerar de la siguiente manera:

- Analizar la legislación española relativa al uso y el desarrollo de las TIC's anterior a la Ley 11/2007, de 22 de junio de Acceso Electrónico de los Ciudadanos a los Servicios Públicos.

- Profundizar sobre dos aspectos de la Ley 11/2007: el derecho de acceso de los ciudadanos a la administración electrónica y el software libre.

- Establecer las características y diferencias entre las licencias de software y el software libre.

- Determinar las implicaciones jurídicas del software libre distinguiendo entre derechos de autor (copyright), copyleft y Licencia Pública General (GPL).

- Demostrar la conveniencia de aplicación y de uso de las licencias libres en la Administración Pública Española.

La metodología aplicada parte de un examen previo de la legislación europea y española relativa a las TIC's y a la implantación del software libre en la Administración Pública. Seguidamente, se enumeran y se establecen las características de las diferentes formas de licenciamiento del software libre para acabar determinando las ventajas de su aplicación para la Administración Pública y para el administrado.

\section{ADMINISTRACIONES PÚBLICAS ELECTRÓNICAS}

\subsection{REGULACIÓN Y PRINCIPIOS BÁSICOS}

(...) las administraciones públicas tienen la tarea de transformar o adaptar su función, organización, formas de actuación y de relacionarse -externa e internamente- a las nuevas exigencias de la realidad, a través de una tecnificación y mo- 
dernización que permita ajustarse a las innovaciones tecnológicas ${ }^{l}$.Ello se debe a que el desarrollo de la sociedad de la información y de las nuevas tecnologías de la información y del conocimiento (TIC's) impone a los poderes públicos la obligación de promover, en beneficio de los administrados, las comunicaciones electrónicas. De este modo, la administración pública electrónica implica un acercamiento del aparato público a los administrados, pues éstos pueden relacionarse electrónicamente las 24 horas del día, los 7 días de la semana y los 365 días del año, sin barreras y restricciones inherentes a los horarios de atención y de servicio.

Por todo ello, podemos definir a las administraciones públicas electrónicas como aquéllas que utilizan de manera extensiva e intensiva las tecnologías de la información y de la comunicación en su organización, funciones o competencias y relaciones internas y externas, con los fines de racionalizar el gasto público, mejorar la calidad de los servicios públicos, obtener mayores grados de eficiencia y eficacia, promover la participación ciudadana, facilitar la rendición de cuentas y la evaluación del desempeño.

Sin embargo, la incorporación de la administración electrónica en las administraciones públicas implica cambios en la organización interna y nuevas aptitudes del personal.

\subsection{BASES JURÍDICAS}

La utilización de las TIC's por parte de las administraciones públicas y el reconocimiento del derecho de los administrados a comunicarse por medios electrónicos con éstas, pone de manifiesto la necesidad de establecer un nuevo régimen jurídico, el cual deberá tener en cuenta el uso extensivo de las TIC's y el reconocimiento efectivo del derecho de los ciudadanos al uso de la tecnología como forma de relacionarse con la administración pública.

Este nuevo régimen jurídico ha de contener una serie de elementos básicos como: la regulación de la sede electrónica, de los registros electrónicos, del expediente electrónico, de las comunicaciones electrónicas, de los archivos electrónicos y de los modos de identificación y autenticación ${ }^{2}$. En el caso español, todos estos puntos quedan definidos y regulados en la Ley 11/2007, de 22 de junio, de Acceso Electrónico de los Ciudadanos a los Servicios Públicos.

\footnotetext{
${ }^{1}$ JINESTA LOBO, Ernesto (2008): “Administraciones públicas electrónicas: retos y desafíos para su regulación”, IVSTITIA, año 22, n 261-262, Isoconsulter, S.A y Editorial Jurídica Continental, San José (Costa Rica), p.4-16.

${ }^{2}$ La suma de todos estos elementos permiten el establecimiento de una oficina administrativa virtual de carácter integral que se encuentre en un sitio web y a la que se pueda acceder a través de un portal.
} 
No obstante, el proceso de modernización administrativa de las administraciones públicas españolas tiene su precedente en la Ley 30/1992, de 26 de noviembre, de Régimen Jurídico de las Administraciones Públicas y del Procedimiento Administrativo Común ( $L P A C)$. Unos de los elementos más destacables de la LPAC es el fomento de las TIC's como forma de mejorar los servicios existentes disminuyendo errores, plazos de resolución o trámites burocráticos junto a la prestación de nuevos servicios. El objetivo de todo ello es mejorar la productividad y la calidad de los servicios gracias a una mayor eficiencia en la gestión de los recursos públicos y una racionalización de los métodos de trabajo ${ }^{3}$.

Analizada la LPAC, se puede comprobar cómo se recogen una serie de disposiciones que aluden al empleo de las TIC's. De este modo:

- Regulación de la organización y de la actividad de los registros administrativos, previendo su informatización (Artículo 38).

- Incorporación de las técnicas automatizadas de la Administración y en sus relaciones con los ciudadanos (Artículo 45).

- Utilización de los medios electrónicos, informáticos y telemáticos (Artículo 59).

- Indicación por parte del administrado de escoger la identificación del medio preferente o del lugar que señale a efectos de notificación (Artículo 70).

- Informatización de los registros (Disposición Adicional 2a).

- Presentación telemática de solicitudes y comunicaciones dirigidas a la Administración General del Estado y sus organismos públicos (Disposición Adicional $18^{\mathrm{a}}$ ).

La regulación de las TIC's se desarrolló en España a partir del Real Decreto 263/1996, de 16 de febrero, por el que se regula la utilización de técnicas electrónicas, informáticas y telemáticas por la Administración General del Estado. De esta reglamentación, quizá lo más interesante sean los siguientes puntos:

- Apartados 1 y 3: La utilización de técnicas y medios en la actuación administrativa, tramitación y terminación de procedimientos administrativos en soporte informático.

- Apartado 4: El desarrollo de los programas y aplicaciones informáticos para el ejercicio de potestades administrativas.

${ }^{3}$ Los puntos básicos de la LPAC fueron recuperados en el año 2000 por el Gobierno Español en el Libro Blanco para la mejora de los servicios públicos. Una nueva Administración al servicio de los ciudadanos. Entre las estrategias recogidas, destacan la de "Mejorar la atención a los ciudadanos y establecer un sistema integral de comunicación con la Administración" $\left(\right.$ Capítulo $8^{\circ}$ ) y la de "Integrar las administraciones públicas en la Sociedad de la Información y del Conocimiento" (Capítulo 10). 
- Apartado 2: La regulación de las relaciones entre el ciudadano y la Administración.

- Apartado 5: La emisión de documentos y copias a través de las TIC's.

Otra norma reglamentaria derivada de la LPAC es el Real Decreto 772/1999, de 7 de mayo, que regula la presentación de solicitudes, escritos y comunicaciones ante la Administración General del Estado, la expedición de copias de documentos y devolución de originales, así como el régimen de las oficinas de registro (RPSEC). Entre los elementos del RPSEC destacan:

- Disposiciones sobre la habilitación de los medios informáticos, electrónicos y telemáticos para la presentación de solicitudes, escritos y comunicaciones (Artículo 3.1b).

- Integración de los modelos de solicitud en sistemas normalizados que permitan la transmisión por medios telemáticos (Artículo 5.2).

- Expedición informática de recibos acreditativos de presentación de escritos (Artículo 6.3).

- Instalación en soporte informático de las oficinas de registro para (...) garantizar la plena conexión e integración de las de carácter general y las respectivas oficinas de carácter auxiliar (Artículo 12.4).

La Ley 6/1997, de 14 de abril, de Organización y Funcionamiento de la Administración General del Estado proclama el principio de servicio a los ciudadanos y prevé que éstos puedan recibir información de interés general por medios telefónicos, informáticos y telemáticos (Artículo 4.2a).

Las leyes y reglamentos anteriormente citados se complementan con una serie de normas de certificación electrónica y de protección de datos aprobadas con el fin de garantizar la seguridad, la validez y la eficacia de la emisión y recepción de comunicaciones y documentos, mediante técnicas y medios electrónicos, informáticos y telemáticos entre las Administraciones Públicas y los administrados. En este sentido son destacables:

- La Ley 66/1997, de 30 de diciembre, de Medidas Fiscales, Administrativas y del Orden Social: En su Artículo 81 se señala a la Fábrica Nacional de Moneda y Timbre como entidad certificadora para garantizar la seguridad y validez de las comunicaciones a través de medios electrónicos, informáticos y telemáticos.

- El Real Decreto-Ley 14/1999, de 17 de septiembre, sobre firma electrónica: En el Artículo 5 se regula el empleo específico de la firma electrónica por parte de las administraciones públicas.

- La Ley Orgánica 15/1999, de 13 de diciembre, de Protección de Datos de Carácter Personal y el Real Decreto 994/1999, de 11 de junio, por el que se aprueba el Reglamento de medidas de seguridad en los ficheros automatizados que contengan datos de carácter personal. 
El conjunto de toda esta normativa y reglamentación jurídica española debe tenerse en cuenta para comprender en desarrollo y aplicación de la Ley 11/2007, de 22 de junio, de Acceso Electrónico de los Ciudadanos a los Servicios Públicos.

\section{LA LEY DE ADMINISTRACIÓN ELECTRÓNICA}

La administración electrónica es calificada por el Dr. Rubén Martínez Gutié$\mathrm{rrez}^{4}$ como (...) un modelo de administrar basado en la aplicación de las tecnologías de la información y la comunicación en el desarrollo de las actividades administrativas con dos dimensiones diferenciadas: de un lado, la dimensión interna, que comprende la aplicación de las TIC's en el trabajo administrativo interno y en las relaciones interadministrativas; $y$, de otro lado, la dimensión externa, referida a la aplicación de las TIC's con el objetivo de ofrecer servicios públicos y procedimientos administrativos en sede electrónica a los administrados.

Partiendo de esta definición y de la base jurídica española anteriormente comentada, la Ley 11/2007, de 22 de junio, de Acceso Electrónico de los Ciudadanos a los Servicios Públicos presenta como elemento fundamental el paso del podrán por el deberán, obligando a las Administraciones a la utilización de los medios electrónicos 5 .

Para ello, la Ley 11/2007 regula las herramientas tecnológicas necesarias para implantar el nuevo modelo de administración como son:

1. La sede electrónica.

2. El registro electrónico.

3. La notificación electrónica.

4. La firma electrónica.

Es obligación de la Administración garantizar el acceso de los ciudadanos a los Servicios Públicos habilitando estos cuatro instrumentos ${ }^{6}$.

${ }^{4}$ MARTÍNEZ GUTIÉRREZ, Rubén (2007): “Administración electrónica: origen, definición institucional y contexto actual", Revista Aranzadi de Derecho y Nuevas tecnologías, $\mathrm{n}^{\circ} 14$, Editorial Aranzadi, Pamplona, p.79-100.

${ }^{5}$ Mediante la Ley 11/2007, desde el 1 de enero de 2010 la Administración electrónica debería ya ser una realidad en España.

${ }^{6}$ Para la definición de estas cuatro herramientas, ver MARTÍNEZ GUTIÉRREZ, Rubén (2009): "Instrumentos para el acceso de los ciudadanos a la Administración electrónica", Derecho, gobernanza y tecnologías en la sociedad del conocimiento, Lefis series, vol.7, $\mathrm{n}^{\circ}$ 9, Universidad de Zaragoza, Zaragoza, p-193-220. 


\subsection{DERECHOS DE LOS CIUDADANOS}

Con la Ley 11/2007 los ciudadanos son el centro de la administración electrónica y verdaderos coprotagonistas de su gestión ${ }^{7}$. Los derechos de los ciudadanos frente a la Ley 11/2007 pueden dividirse en dos grandes bloques: el derecho de los ciudadanos a relacionarse electrónicamente con la administración y los derechos jurídicos del administrado electrónicamente ${ }^{8}$.

La Ley 11/2007 indica que los ciudadanos tienen el derecho a relacionarse electrónicamente con la administración así como para obtener informaciones, realizar consultas y alegaciones, formular solicitudes, manifestar consentimiento, entablar pretensiones, efectuar pagos, realizar transacciones y oponerse a las resoluciones y actos administrativos (Artículo 6.1 ${ }^{\circ}$ ).Ello implica que la Administración debe asegurarse que toda persona pueda relacionarse electrónicamente con ella a través de los canales que establezca. Por este motivo, no sólo se deben habilitar los canales, sino también asegurarse que ningún ciudadano quede impedido a su acceso?.

Respecto al segundo grupo de derechos, éstos son una derivación del Artículo $35 \mathrm{f}$ ) de la Ley 30/1992 en el que se indica que el administrado tiene derecho (...) a no presentar documentos no exigidos por las normas aplicables al procedimiento de que se trate, o que ya se encuentren en poder de la Administración actuante. Mediante el Artículo $6.2^{\circ}$ b) de la Ley $11 / 2007$, este derecho no se limita ya a la administración actuante, sino que es extensible para todas las administraciones ${ }^{10}$.

Si se analizan todos los puntos del Artículo 35 de la Ley 30/1992 y los comparamos con los del Artículo 6.2 $2^{\circ}$ de la Ley 11/2007, se podrán observar otros puntos convergentes. En este sentido, se pueden enumerar:

${ }^{7}$ COTINO HUESO, Lorenzo (2008): "Los derechos de los ciudadanos ante la administración electrónica", dentro de proyecto De la administración al gobierno electrónicos; régimen e implicaciones jurídicas y constitucionales, Ed. Tirant lo Blanch, Valencia, p.1-30, p.4.

${ }^{8}$ COTINO HUESO, LORENZO (2008): "Derechos del ciudadano" en La Ley de Administración Electrónica. Comentario sistemático a la Ley 11/2007, de 22 de junio, de Acceso Electrónico de los Ciudadanos a los Servicios Públicos, coordinada por Eduardo Gamero Casado y Julián Valero Torrijos, Editorial Aranzadi, Pamplona, p.117-233.

${ }^{9}$ Por ello, el Artículo 8 impone el establecimiento de oficinas de atención presencial, puntos de acceso electrónico o sedes electrónicas.

${ }^{10}$ Este derecho es una consecuencia de la implantación de las TIC's que da lugar a un cambio de modelo de Administración por un modelo de administración electrónica de intercambiabilidad de datos sin intervención directa de órganos ni personal administrativo en los procesos de comunicación. 
- Derecho del administrado a conocer electrónicamente el estado de los procedimientos, electrónicos o no y derecho a obtener copias electrónicas de su propio expediente (Artículo $6.2^{\circ} d$ ).

- Derecho a la calidad de los servicios públicos prestados por medios electrónicos (Artículo 6.2 $\mathrm{j}$ ).

- Derecho de elección de canales, las aplicaciones o sistemas a través de los cuales pueda relacionarse por medios electrónicos con la Administración (Artículo 6.2 $a$ ).

- Derechos a obtener y usar medios de identificación (Artículo $6.2 \mathrm{~g}$ y $h$ ).

- Derecho a la conservación de los documentos en formato electrónico por la Administración (Artículo 6.2f).

- Derecho a la garantía de la seguridad y confidencialidad de los datos que figuren en los ficheros, sistemas y aplicaciones de las Administraciones públicas (Artículo 6.2 i).

- Derecho a obtener información específica sobre el establecimiento de actividades de servicios (Artículo 6.3).

\subsection{EL SOFTWARE LIBRE}

Junto a todo lo analizado en el punto anterior, hay un elemento más a destacar y es la relación entre la Ley 11/2007 y los derechos de los ciudadanos ante la administración electrónica y el uso de licencias de software libre por parte de la Administración.

En este sentido, en el artículo $6.2^{\circ} \mathrm{k}$ ) se reconoce el derecho: $A$ elegir las aplicaciones o sistemas para relacionarse con las Administraciones públicas siempre y cuando utilicen estándares abiertos o, en su caso, aquellos otros que sean de uso generalizado por los ciudadanos. Mientras que el artículo $6.2^{\circ} \mathrm{c}$ ) reafirma el de la igualdad en el acceso electrónico a los servicios de las Administraciones públicas.

Los derechos de los ciudadanos se han continuado consolidando tras la Ley 11/2007 mediante el Real Decreto 4/2010, de 8 de enero, por el que se regula el Esquema Nacional de Interoperabilidad en el ámbito de la Administración Electrónica.

Según se indica en él, la Administración tiene la obligación de:

- Promover las condiciones para que la libertad y la igualdad de acceso sean reales y efectivas.

- Eliminar los obstáculos que impidan o dificulten el ejercicio pleno del principio de neutralidad tecnológica.

- Garantizar la independencia en la elección de las alternativas tecnológicas por los ciudadanos.

- Promover la libertad de desarrollar e implantar los avances tecnológicos en un ámbito de libre mercado. 
Junto a todo ello, el Real Decreto 4/2010 dedica el Capítulo V a la Interoperabilidad técnica. El Artículo 11 de dicho capítulo está dedicado a los estándares aplicables mostrando claramente su inclinación hacia las licencias de software libre: (...) Las Administraciones públicas usarán estándares abiertos, así como, en su caso y de forma complementaria, estándares que sean de uso generalizado por los ciudadanos, al objeto de garantizar la independencia en la elección de alternativas tecnológicas por los ciudadanos y las Administraciones públicas y la adaptabilidad al progreso de la tecnología (...).

Sin embargo, el Real Decreto 4/2010 no sólo se detiene ahí, sino que limita el uso en exclusiva del estándar no abierto a aquellos casos en los que (...) no se disponga de un estándar abierto que satisfaga la funcionalidad satisfecha por el estándar no abierto en cuestión y sólo mientras dicha disponibilidad no se produzca.

Atendiendo a estos derechos de los ciudadanos y a las obligaciones respecto al administrado, la Administración debe elegir aplicaciones o sistemas que utilicen estándares abiertos, es decir, que estén disponibles y gratuitos a un costo que no suponga dificultades para su acceso, o aquéllos que sean de uso general y común por la Ciudadanía y que puedan garantizar la homogeneidad de las herramientas tecnológicas, la interoperabilidad y la compatibilidad con las plataformas informáticas de las administraciones públicas. Ello significa que el uso de las licencias libres se presentan para la Ley 11/2007 como una primera opción en el ámbito de la Administración pública como forma de garantizar el intercambio de datos entre administraciones y entre administraciones y administrados. A la vez, se presenta como una garantía de defensa del derecho del ciudadano a no ser discriminado tecnológicamente.

\section{LAS LICENCIAS DE SOFTWARE Y SU MARCO JURÍDICO}

\subsection{MODELOS DE LICENCIAMIENTO DEL SOFTWARE}

El marco jurídico español no estipula una fórmula legal específica para la cesión de los derechos de autor de software a terceros hecho por el cual se utiliza habitualmente el contrato para el software de licencia.

No obstante, hay otro tipo de software que se conoce como software libre. Éste se puede definir como aquel software distribuido bajo un determinado tipo de licencia de uso que permite el acceso al código objeto y al código fuente del mismo, y por tanto su modificación y adaptación a las necesidades del usuario, y 
también la libre distribución y utilización de la aplicación ${ }^{11}$. En ningún caso, debe entenderse como software gratuito.

Partiendo de esta segunda tipología de software, Jordi Mas i Hernàndez ${ }^{12}$ establece tres modelos de licenciamiento atendiendo a los derechos que ceden parte de los autores a los usuarios y bajo qué condiciones. De este modo:

1. Licencias con copyflet: Son aquellas que ceden los derechos de copia, distribución y modificación del programa bajo las condiciones que definen el software libre, pero que además exigen que cualquier versión modificada herede el mismo tipo de obligaciones y derechos que tenía el programa original con la finalidad de garantizar que cualquier usuario conserve en el futuro las libertades originales.

2. Licencias de código abierto o permisivas: Este tipo de licencias ceden el uso del programa bajo las condiciones que definen el software libre, pero no obligan a hacer públicas las mejoras realizadas sobre el código fuente.

3. Licencias duales: En este modelo el autor cede su creación bajo dos licencias diferentes según el uso que se vaya a hacer de su software y de las libertades y obligaciones que se deseen adquirir.

\subsection{SOFTWARE LIBRE Y SUS IMPLICACIONES JURÍDICAS: DERECHOS DE AUTOR (COPYRIGHT), COPYLEFTY LICENCIA PÚBLICA GENERAL (GPL $\left.{ }^{13}\right)$}

Para Gladis Stella Rodríguez ${ }^{14}$, el software libre es una oportunidad para democratizar el acceso a la información.

Sin embargo para la Organización Mundial de la Propiedad Intelectual (OMPI), el software libre es en sí mismo una forma de ejercicio del derecho de autor ${ }^{15}$ debido a que:

1. El autor del software es legalmente el titular del mismo.

${ }^{11}$ AAVV (2004): Aspectos legales y de explotación del software libre. Parte I, Universitat Oberta de Catalunya, Barcelona, p.44-45.

${ }^{12}$ MAS I HERNÀNDEZ, Jordi (2005): Software libre: técnicamente viable, económicamente sostenible y socialmente justo, Zero Factory, S.L., Barcelona, p.70-74.

${ }^{13}$ Acrónimo inglés de Licencia Pública General.

${ }^{14}$ RODRÍGUEZ, Gladys Stella (2008): "El software libre y sus implicaciones jurídicas", Revista de Derecho, $\mathrm{n}^{\circ}$ 30, diciembre 2008, Universidad del Norte, Colombia, p.164-169.

${ }^{15}$ Tratado de la OMPI sobre Derechos de Autor (TODA) entró en vigor el 6 de marzo de 2002 con el fin de proteger a los autores de obras literarias y artísticas, incluyendo programas informáticos y bases de datos originales. 
2. El autor mantiene los derechos de reproducción y distribución del programa, así como los que permiten su modificación para poder garantizar la continuidad del software libre mediante los principios de libertad de uso, libertad de adaptación, libertad de distribución y libertad de mejorarlo.

En consecuencia, se puede afirmar que el software libre está sometido a las leyes de derecho de autor con el objetivo de garantizar las libertades originales ya sea mediante el uso del copyright, del copyleft o de la Licencia Pública General (GPL).

El derecho de autor es un término jurídico que describe los derechos concedidos a los creadores por sus obras literarias, artísticas y científicas entre los que se incluyen los programas informáticos. Tradicionalmente en Europa continental y en España, el término copyright se utiliza como sinónimo de derecho de autor ${ }^{16}$.En el marco jurídico europeo, los derechos de autor y los programas de ordenador se rigen por la Directiva 91/250/CEE del Consejo de 14 de mayo de 1991 relativa a la protección jurídica de programas de ordenador y por la Directiva 93/98/CEE del Consejo de 29 de octubre de 1993. En ambas se establece que los programas de ordenador han de protegerse por los derechos de autor como si fuesen obras literarias siguiendo los principios de la Convención de Berna ${ }^{17}$.

Estas directivas europeas se complementan con la Directiva 2001/29/CEE, de 22 de mayo de 2001 relativa a la armonización de determinados aspectos de los derechos de autor y derechos afines a los derechos de autor en la sociedad de la información ampliando, así, los conceptos de reproducción y de comunicación pública que se aplican tanto al software como a la documentación complementaria distribuida por Internet.

En el ámbito jurídico español, la regulación de la propiedad intelectual tiene su precedente en los artículos 428 y 429 del Código civil español. Con posterioridad, la Ley de Propiedad Intelectual (LPI) ${ }^{18}$, el Real Decreto Legislativo $1 / 1996^{19}$ y la

\footnotetext{
${ }^{16}$ En la tradición jurídica anglosajona, el término copyright se basa en la idea de que lo más importante no es el autor, sino la obra y los derechos de propiedad que los autores tienen sobre sus creaciones. Se podría traducir como "derecho de copia".

${ }^{17}$ El año 1886 tuvo lugar el Convenio Universal de Ginebra para la Protección de Obras Literarias y Artísticas siendo su última revisión en el año 1971.

${ }^{18}$ Ley 22/1987, de 11 de noviembre, de Propiedad Intelectual. Actualmente derogada tras la aprobación del Real Decreto Legislativo 1/1996.

${ }^{19}$ Real Decreto Legislativo 1/1996, de 12 de abril, por el que se aprueba el texto refundido de la Ley de Propiedad Intelectual, regularizando, aclarando y armonizando las disposiciones legales vigentes sobre la materia.
} 
Ley $23 / 2006^{20}$ continúan equiparando los programas de ordenador (software) a las obras literarias protegiéndolos por el sistema de los derechos de autor.

No obstante, la protección del software por derechos de autor presenta dos características a tener en cuenta: 1 . Únicamente se protege la expresión del programa de ordenador y no, las ideas que contiene; 2 . Solamente se protege al software como bien inmaterial, independientemente del soporte en el cual se fije.

En consecuencia, los derechos de autor protegen los siguientes elementos del software: el programa de ordenador propiamente dicho, la documentación preparatoria, los manuales de uso y documentación técnica de apoyo, el código fuente, el código objeto, la arquitectura del programa, las interfaces y las bases de datos.

Atendiendo al artículo 10.1 de la LPI, en el que se indica que las obras son aquellas creaciones humanas originales expresadas en cualquier medio o soporte, se puede afirmar que el software puede ser protegido porque cumple los tres requisitos: es de creación humana, se expresa en un medio o soporte informático y es original.

Según Richard M. Stallman ${ }^{21}$, el copyleft se puede definir como el régimen jurídico que salvaguarda el dominio público del software libre y evita que pueda ser convertido en software propietario por los usuarios. De esta manera, el copylef permite licenciar el software libre de tal forma que su uso y modificación permanezcan siempre libres evitando el monopolio legal de explotación, aunque carece de reconocimiento legal. Mediante el copyleft se da a cualquier persona el permiso para usar el programa, copiarlo, modificarlo y redistribuir versiones nuevas; pero no, para agregar restricciones propias. En consecuencia, las versiones modificadas del software libre también deben ser libres.

La GPL es la licencia más conocida en el mundo del software libre. Este tipo de licencia pretende garantizar la libertad de compartir y modificar el software libre más allá del ámbito contractual inmediato, asegurando su libertad para usuarios posteriores. Con ello se garantiza la libertad del código en todo momento, ya que todo software publicado y licenciado bajo las condiciones de la GPL nunca podrá ser software propietario.

${ }^{20}$ Ley 23/2006, de 7 de julio, por la que se modifica el texto refundido de la Ley de Propiedad Intelectual, aprobado por el Real Decreto Legislativo 1/1996, de 12 de abril.

${ }^{21}$ Richard Matthew Stallman es conocido por el establecimiento de un marco de referencia para el movimiento del Software libre como alternativa al desarrollo y distribución del software no libre o privativo y por ser el inventor del concepto copyleft (aunque no, del término). 


\section{SOFTWARE LIBRE EN EL SECTOR PÚBLICO}

La Carta Iberoamericana de Gobierno Electrónico de Pucón de 1 de junio de $2007^{22}$ establece una serie de principios entendidos como obligaciones administrativas para la implantación de la administración electrónica en los gobiernos iberoamericanos. El más interesante para este estudio es el Principio de adecuación tecnológica, según el cual las administraciones públicas tienen la obligación de elegir las tecnologías más adecuadas para satisfacer sus necesidades y la de los administrados.

Por este motivo, en dicha carta se recomienda el uso de estándares abiertos y de software libre atendiendo a tres premisas: seguridad, sostenibilidad a largo plazo y prevención ante una privatización del conocimiento público.

A éste se le añade el Principio de neutralidad tecnológica y adaptación al progreso de las técnicas y sistemas de comunicación electrónicas mediante el cual los entes públicos deben garantizar la independencia en la elección de las alternativas tecnológicas escogidas por éstos y los administrados, así como la libertad de desarrollar e implantar los avances tecnológicos en un ámbito de libre mercado. Para ello, las administraciones deben utilizar estándares abiertos o estándares que sean de uso generalizado.

\subsection{CONVENIENCIAS DEL USO DEL SOFTWARE LIBRE}

Para el Centro Nacional de Referencia de Aplicación de las Tecnologías de la Información y la Comunicación (CENATIC), la verdadera innovación que presenta el software libre es la plena accesibilidad del código, su libertad de uso y de copia, así como la posibilidad de mejora compartida y colaborativa por parte de la comunidad de desarrolladores y programadores que trabajan en él ${ }^{23}$.

El uso del software libre presenta una serie de ventajas para la Administración y garantiza los derechos de los administrados establecidos en la Ley 11/2007 entre las cuales, se pueden enumerar:

\footnotetext{
${ }^{22}$ Aprobada en la IX Conferencia Iberoamericana de Ministros de Administración Pública y Reforma del Estado.

${ }^{23} \mathrm{AAVV}$ (2008): Guía básica del software de fuentes abiertas, CENATIC, Almendralejo, p.8.
} 
1. El Coste: El software libre no tiene prácticamente coste de licencia hecho que le permite invertir parte del presupuesto en mejoras adaptadas a las necesidades de cada administración y en formación del personal ${ }^{24}$.

2. La Innovación tecnológica: En el modelo de software libre prima el hecho de compartir la información y el trabajo cooperativo.

3. La Independencia del proveedor gracias a la disponibilidad del código fuente. Cualquier empresa o profesional puede seguir ofreciendo desarrollo o servicio de mantenimiento a la aplicación.

4. El desarrollo de la industria local: En el software libre no hay coste de licencia debido al derecho de copia y al disponer del código fuente, permite el desarrollo interno de mejoras o modificaciones sin necesidad de encargarlas a empresas fuera del ámbito local.

5. La seguridad y privacidad de los datos personales: El software libre, al disponer del código fuente, mejora diversos aspectos relacionados con la perennidad de los datos y su seguridad.

6. La adaptación del software: El software libre permite personalizar los programas hasta cubrir las necesidades particulares de cada Administración.

7. La lengua: El software libre puede adaptarse a la lengua minoritaria escogida y se puede desarrollar o adaptar un corrector lingüístico ${ }^{25}$.

\section{CONCLUSIONES}

A modo de síntesis, se pueden extraer una serie de ideas clave:

1. La Administración Pública tiene que ser responsable a la hora de adquirir TIC's, ya que opera con dinero público. Ello implica que los gastos deben estar muy estudiados y prevaleciendo siempre la relación calidad-coste. El software libre, al no tener costes de licencia y al tener el código fuente abierto, permite reducir los costes de implantación en la Administración ya que cualquier desarrollador de software puede ponerlo en funcionamiento y adaptarlo a sus necesidades particulares.

2. Desde la Ley 11/2007, la Administración debe garantizar el acceso de los ciudadanos a los Servicios Públicos. Ello tiene dos implicaciones fundamentales:

a. Los ciudadanos tienen el derecho a relacionarse electrónicamente con la Administración.

\footnotetext{
${ }^{24} \mathrm{El}$ análisis TCO (Total Cost of Ownership) permite conocer el coste total de la propiedad que tiene una determinada solución de software reflejando no sólo el coste, sino también la ayuda y el mantenimiento tecnológico de la solución.

${ }^{25}$ Este punto es interesante en el caso español atendiendo a aquellas Comunidades Autónomas con cooficialidad lingüística en la Administración y entre la Ciudadanía.
} 
b. La Administración debe garantizar el acceso electrónico de los ciudadanos a los Servicios Públicos electrónicos prevaleciendo el uso de licencias de software libre para asegurar la igualdad de acceso electrónico a la Administración evitando, así, la discriminación tecnológica.

3. Con el fin de asegurar el acceso al código fuente, el software libre está sometido a las leyes de derecho de autor, ya sea mediante el copyright, el copyleft o la Licencia Pública General (GPL).

4. El uso del software libre por parte de la Administración previene la privatización del conocimiento público ya que la información no puede quedar monopolizada por una licencia o un servidor de software pues el código de acceso que permite su desarrollo es abierto.

De todo ello, se puede afirmar que las licencias de software libre aúnan en sí mismas estos dos puntos: un gasto responsable y una no vulneración del derecho del administrado a acceder a la administración pública electrónica determinando, por tanto, que la Ley 11/2007 prima la aplicación de las licencias libres frente a las licencias privadas.

\section{Legislación consultada:}

Legislación europea:

Directiva 91/250/CEE del Consejo de 14 de mayo de 1991.

Directiva 93/98/CEE del Consejo de 29 de octubre de 1993.

Directiva 2001/29/CEE, de 22 de mayo de 2001.

Legislación española:

Código civil español.

Ley 30/1992, de 26 de noviembre, de Régimen Jurídico de las Administraciones Públicas y del Procedimiento Administrativo Común.

Ley 6/1997, de 14 de abril, de Organización y Funcionamiento de la Administración General del Estado.

Ley 66/1997, de 30 de diciembre, de Medidas Fiscales, Administrativas y del Orden Social.

Ley 23/2006, de 7 de julio, por la que se modifica el texto refundido de la Ley de Propiedad Intelectual, aprobado por el Real Decreto Legislativo 1/1996, de 12 de abril.

Ley 11/2007, de 22 de junio, de Acceso Electrónico de los Ciudadanos a los Servicios Públicos.

Ley Orgánica 15/1999, de 13 de diciembre, de Protección de Datos de Carácter Personal y el Real Decreto 994/1999, de 11 de junio, por el que se aprueba el Reglamento de medidas de seguridad en los ficheros automatizados que contengan datos de carácter personal. 
Real Decreto 263/1996, de 16 de febrero, por el que se regula la utilización de técnicas electrónicas, informáticas y telemáticas por la Administración General del Estado.

Real Decreto 772/1999, de 7 de mayo, que regula la presentación de solicitudes, escritos y comunicaciones ante la Administración General del Estado, la expedición de copias de documentos y devolución de originales, así como el régimen de las oficinas de registro.

Real Decreto 4/2010, de 8 de enero, por el que se regula el Esquema Nacional de Interoperabilidad en el ámbito de la Administración Electrónica.

Real Decreto Legislativo 1/1996, de 12 de abril, por el que se aprueba el Texto Refundido de la Ley de Propiedad Intelectual, regularizando, aclarando y armonizando las disposiciones legales vigentes sobre la materia.

\section{REFERENCIAS BIBLIOGRÁFICAS}

Aspectos legales y de explotación del software libre. Parte I. Barcelona: Universitat Oberta de Catalunya, 2004.

COTINO HUESO, LORENZO. "Derechos del ciudadano", La Ley de Administración Electrónica. Comentario sistemático a la Ley 11/2007, de 22 de junio, de Acceso Electrónico de los Ciudadanos a los Servicios Públicos, Pamplona: Editorial Aranzadi, 2008, pp.117-233.

COTINO HUESO, Lorenzo. "Los derechos de los ciudadanos ante la administración electrónica", De la administración al gobierno electrónicos; régimen e implicaciones jurídicas y constitucionales, Valencia: Ed. Tirant Lo Blanch, 2010, pp.1-30.

Guía básica del software de fuentes abiertas. Almendralejo: CENATIC, 2008.

JINESTA LOBO, Ernesto. "Administraciones públicas electrónicas: retos y desafíos para su regulación”, IVSTITIA, año 22, n 261-262, San José (Costa Rica): Isoconsulter, S.A y Editorial Jurídica Continental, 2008, pp.4-16.

Libro Blanco para la mejora de los servicios públicos. Una nueva Administración al servicio de los ciudadanos, Madrid: Ministerio de Administraciones Públicas, 2000.

MARTÍNEZ GUTIÉRREZ, Rubén. “Administración electrónica: origen, definición institucional y contexto actual", Revista Aranzadi de Derecho y Nuevas tecnologías, $\mathrm{n}^{\mathrm{o}}$ 14, Pamplona: Ed. Aranzadi, 2007, pp.79-100.

MARTÍNEZ GUTIÉRREZ, Rubén. "Instrumentos para el acceso de los ciudadanos a la Administración electrónica", Derecho, gobernanza y tecnologías en la sociedad del conocimiento, Lefis series, vol. 7, $\mathrm{n}^{\circ}$ 9, Zaragoza: Universidad, 2009, pp.193-220.

MAS I HERNÀNDEZ, Jordi. Software libre: técnicamente viable, económicamente sostenible y socialmente justo, Barcelona: Zero Factory, S.L., 2005

RODRÍGUEZ, Gladys Stella. "El software libre y sus implicaciones jurídicas", Revista de Derecho, $\mathrm{n}^{\mathrm{o}}$ 30, diciembre 2008, Colombia: Universidad del Norte, pp.164-169. 